\title{
XII.
}

\section{Multiple Neurome im Bereich des Plexus brachialis sinister, cavernöse Angiome, Lymphangiome und Neurofibrome der linken oberen Extremität.}

\author{
Von Prof. Heinrich Köbner in Berlin.
}

(Hierzu Taf. VI.)

Der nachfolgende Fall') bietet eine seltene Combination von, auf Bildungsanomalien an den Nerven des Plex. brachial. sin. in seiner gesammten, auch thoracischen Ausbreitung und an den Gefässen der linken oberen Extremität beruhenden Geschwülsten dar in Form von multiplen Neuromen, cavernösen Angiomen, Lymphangiomen und neuromatösen Fibromen der Haut und des Unterhautgewebes. Der Träger derselben hatte sich im März dieses Jahres nur behufs Heilung einer alten Acne indur. der Nase an mich gewandt, aber alsbald fesselten die überaus seltsamen Tumoren und Deformitäten seiner linksseitigen Finger mein Augenmerk und veranlassten mich zu eingehender Gesammtuntersuchung.

B. Z., Kaufmannssohn aus einer Stadt der Provinz Posen, 21 Jahre alt, ist für sein Alter etwas schwächlich, 5 Fuss 3 Zoll hoch, geistig ziemlich gut entwickelt, nur etwas träge und vergesslich, von hellweisser Hautfarbe, mit reichlichem blondem Kopfhaar, dünnem ebensolchem Schnurrbart, mit graublauer Iris, deren linke im inneren Drittheil ockerfarbig ist.

Der Schädel bietet keine Asymmetrie, die Wirbelsäule eine mässige Scoliose nach rechts, am stärksten im 8. und 9. Brustwirbel, nebst mässiger Lordose, am deutlichsten am 11. und 12. Brust- und 1. Lendenwirbel. Die linke Scapula ist flacher, steht viel weniger hervor, als die rechte, besonders eingefallen ist die Musculatur der Foss. infraspinata, demnächst sind die $\mathrm{Mm}$. longissimus dorsi und rhomboidei schwächer entwickelt (vgl. Fig. 2). Am auffallendsten ist, wenn man den Kranken von vorn betrachtet, auf den ersten Blick die Atrophie des linken Oberarms, die von der Mitte desselben bis auf den Handrücken strotzend, zum Theil geflechtähnlich entwickelten Venen und vor Allem eine Anzahi bläulich- oder gelblich-rother Tumoren

1) Vorgestellt der Berliner med. Gesellschaft am 4. April 1883. 

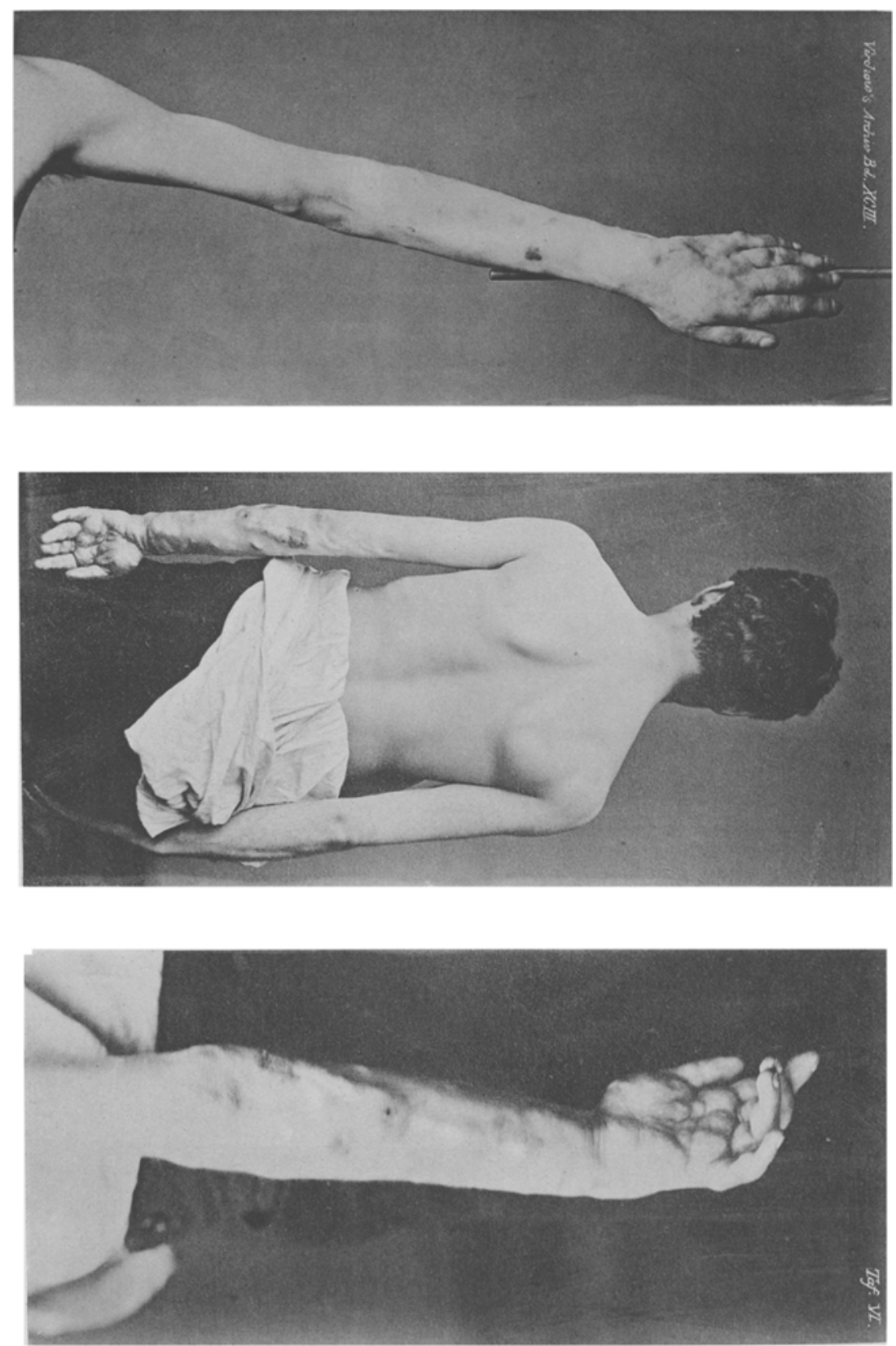
hauptsächlich an der Streckseite des Vorderarms und röthlicher sowie rostbrauner an derjenigen aller Finger; zwischen jenen am Vorderarm liegen einzelne' farblose, andere von angiomatösem Aussehen, ausserdem einzelne, weit auseinander stehende Bläschengruppen auf bräunlichen Flecken oder dicht neben grösseren Angiomen oder neben miliaren angiomatösen Flecken und Punkten. Ferner contrastirt die weisse Grundfarbe der linken, bedeutend kleineren und kürzeren gegenüber der ein wenig livid rothen der rechten Hand. Auch erscheinen der linke Mittel- und 4. Finger, welche auch an den Volarseiten mit Geschwülsten bedeckt sind (Fig. 2), eigenthümlich deform, gegen die Nagelphalanx sich spindelförmig verjüngend, der Mittelfinger im 1. Phalangealgelenk durch je einen kleinen harten Tumor an der Beugeseite seiner 1. und 2. Phalanx ein wenig in Beugungscontractur und mit dem Nagelende nach aussen, der 4. Finger an der Innenseite der 2. Phalanx leicht ausgehöhlt, mit dem Nagelende etwas nach einwärts gerichtet; in jene Aushöhlung, die vor einem grösseren Tumor an der Streckseite und hinter einem kleinen an der Beugeseite dieser 2. Phalanx liegt, passt genau der grösste aller Fingertumoren, derjenige am Dorsum der 2. Phalanx des Mittelfingers (vergl. Fig. 1). -

Der Angabe der gesunden, etwas in sich gekehrten Mutter nach sind in deren Familie keine ähnlichen Geschwülste bekannt, ebenso wenig wie an ihren anderen Söhnen und ihrer Tochter. Auch in Bezug auf functionelle Gehirn- oder Rückenmarkstörungen ist nichts bekannt, als dass je ein älterer Bruder und Schwester der Frau zuweilen an Aufregungszuständen, im Allgemeinen aber an einer gewissen Schwermuth leiden. Bei der Frau selbst erklärt sich diese durch den frühzeitigen Ertrinkungstod ihres Gatten, als unser Patient 3 Jahre alt war, und den Verlust ihrer Tochter, die geistig viel reger war, als jener, sowie dessen 23- und 18jährige Brüder, die sämmtlich etwas träge sind und "schlecht gelernt" haben. Ueber die väterliche Seite ist nichts Sicheres zu eruiren. Geschwülste hatte der Vater, der als leichtlebig geschildert wird, bestimmt nicht. Von den Tumoren am Vorderarme unseres Pat. soll bei der Geburt nur Einer (in der Mitte der Streckseite, der in Folge eines bald zu nennenden Eingriffes nur noch als braunrothe Narbe existirt,) als "blaue Beere" sichtbar gewesen, alle anderen, auch jene an den Fingern, binnen 6 Monaten nach der Geburt bemerkt worden und allmählich gewachsen sein. Soweit Hr. Dr. Ludwig Rosenthal, der den Pat. vor 10 Jahren sah, sich erinnern kann, sind damals die Tumoren am Handrücken, welche jetzt von einem strotzenden Venenconvolut bedeckt sind, noch frei sichtbar gewesen. Die etwas livid rothe Farbe der gesunden Hand soll erst seit 3 Jahren durch Erfrierung aufgetreten sein. -

Um in dem höchst mannichfaltigen Bilde, welches durch die angedeuteten Flecken, Bläschen, Geschwülste und Deformitäten erzeugt wird, uns zu orientiren, beginnen wir mit 2 braunrothen, narbenähnlichen, immer noch derben, 50-Pfennigstück grossen Flecken in der Mitte der Streckseite des Vorderarms und einem ebensolchen über dem Handgelenk, welche aus, vor 10 Jahren durch Electrolyse (galvanische Acupunctur) partiell verödeten 
Angiomen erübrigen, ferner mit mehreren cavernösen, bläulichrothen Angiomen von etwa Markstückgrösse und schwammiger, placentaähnlicher Consistenz vorwiegend an der Dorsal - und an der Volarseite des Vorderarms, deren oberstes, nahe unter dem Condyl. intern. humeri gelegenes erst seit etwa 5 Jahren zu einer über die Haut flach prominirenden, beinahe Dreimarkstückgrossen Gefässgeschwulst geworden, früher ein flaches Gefässmal gewesen sein soll.

Demnächst erkennen wir in den kleinen, Herpes-ähnlichen Bläschengruppen, die zum Theil auf oder dicht an der Grenze jener grösseren oder combinirt mit kleinsten, punktförmigen Hämatangiomen, selten auf einem kleinen braunen, oberflächlich verdickten Fleck, am seltensten auf und, wie man bei Faltung und Durchleuchtung einer davon bedeckten Hautpartie erkennt, als Lücken in derselben bildendes, transparentes, durch feinste Septa getrenntes Fachwerk ans ganz normaler Haut sich erheben und deren klare Lymphe sich während meiner 14tägigen. Beobachtung nicht getrübt, nur über einem grossen Angiom blutig gefärbt hat, ampulläre Erweiterungen der Lymphgefässcapillaren der Haut [Varices der Lymphgefässe der Cutis nach Gjorgjevic ${ }^{1}$, einfache Lymphangiome nach G. Wegner ${ }^{2}$ )].

Für die schwierige Deutung der in ihren Formen sehr bizarren weiterhin näher zu beschreibenden Geschwülste an der Rückseite sämmtlicher Finger aber dient uns die sehr auffallende Schmerzhaftigkeit beim Anfassen einiger plexiform mit einander verbundener, harter Knoten, die unter der diffusen, scheinbar nur varicösen Venenschwellung in der äusseren Hälfte des Handrückens hindurch zu fühlen sind, sowie einiger isolirter farbloser Knoten am Vorderarm als Leitstern. In der Vorstellung, dass dieselben Neurome seien, wird der Plexus cervical. und brachial. sin. abgetastet und an der Portio supraclavicular. des letzteren, besonders vor dem vorderen Rande des M. cucullaris, mehrere parallele Reihen von zahlreichen, spindelförmigen, von $\frac{3}{4}$ bis $3 \mathrm{~cm}$ langen und bis $1 \frac{1}{2} \mathrm{~cm}$ breiten Auftreibungen gefühlt, die sich von etwaigen Lymphdrüsen ausser durch ihr nur linksseitiges Vorkommen theils durch ihren, an einigen deutlich fühlbaren directen Uebergang in Nervenstränge, deren Verlauf von nahe ihren Austrittsstellen nach ab- und auswärts bis nahe zur Clavicula sie folgen, theils

1) Arch. f. klin. Chirurgie. Bd. XII. S. 693.

2) Ebenda Bd. XX. S. 641. - Vgl, auch die Arbeit von P. zur Nieden: Ueber einen Fall von Lymphangiectasie mit Lymphorrhagie (dieses Archiv Bd.90. 2. S. 350 ff.), welcher auf Taf. VI Fig. 1 einen senkrechten Schnitt durch die Cutis einer, mit zahlreichen ebensolchen Bläschen besetzten grossen Schamlefze abbildet. In den tieferen Schichten der Cutis sieht man Querschnitte dickwandiger Lymphgefässe, höher oben, im subpapillären Theil die wandungslosen ectasirten Lymphräume und schliesslich, theilweise in der Epidermis gelegen, mehrere als Blasen makroskopisch sichtbar gewesene Ectasien. 
durch ihre nur geringe, nur nach den Seiten ein wenig mögliche Verschiebbarkeit unterscheiden. Deutlicher noch treten diese Eigenschaften, namentlich ihr Zurückschnellen in die alte Lage beim Loslassen (nach Art eines Knotens in einem gespannten Faden) an 2 spindelförmigen, sich theilweise deckenden Anschwellungen an dem (leichter abhebbaren) Ramus anter. vom Nerv. cutan. lateral. im 4. Intercostalraum, $1 \frac{1}{2} \mathrm{~cm}$ nach aussen von der linken Mammilla hervor, an welche sich auf eine kurze Strecke von $1 \frac{1}{2} \mathrm{~cm}$ normaler Nerv anschliesst, um dann wieder in mehrere linsengrosse, rosenkranzförmige Anschwellungen überzugehen. Von der Achsellinie ab ist der Nerv als normal nach hinten zu verfolgen. In senkrechter Richtung darauf, dicht neben mässig ectatischen Venen, die an der linken Thoraxseite von oben nach abwärts ziehen, fühlt man an den $\mathrm{Nn}$. thoracici I und II sowie thoracico-brachiales ganz dünne Anschwellungen in grösserer Ausdehnung. Vom oberen Rand der 8. bis zur Mitte der 10. linken Rippe beginnt in der hinteren Axillarlinie eine allmähliche, bei flacher Betastung einem jungen Lipom ähnliche, $7 \frac{1}{2} \mathrm{~cm}$ lange und $4 \mathrm{~cm}$ hohe, flache Hervorwölbung der Haut (vergl. Fig. 2), die sich bei tieferem Fingerdruck und beim Abheben in eine grössere Zahl von scharf umschriebenen, spindelförmigen, vorn bis $1 \mathrm{~cm}$ dicken und reichlich bohnengrossen, nach hinten an Grösse abnehmenden Nervenanschwellungen, die untereinander plexiform zusammenzuhängen scheinen, auflösen lässt. Die Haut über allen diesen siubcutanen Neuromen am Thorax ist ganz normal.

Auf der Beugeseite des Oberarms beginnt $5 \mathrm{~cm}$ oberhalb der Ellenbeuge, am Innenrand des Biceps ganz oberflächlich gelegen, das oberste, durch die normale, dünne Haut hellbläulich durchschimmernde, aus 4 kleineren runden zusammengesetzte, fast $1 \mathrm{~cm}$ hohe und $2 \mathrm{~cm}$ lange, beim Emporheben höchst schmerzhafte Convolut von Neuromen am N. cutaneus medius, das durch einen $1 \frac{1}{2} \mathrm{~cm}$ langen, dünnen Verbindungsfaden mit einem solitären, nach ab- und einwärts (nach dem Condyl. intern. humeri hin) gelegenen, erbsengrossen, ebenso durchschimmernden, mässig derben kugligen Neurom zusammenhängt. Demnächst fühlt man ebenda in der Tiefe am $\mathrm{N}$. medianus mehrere, bis etwa bohnengrosse, am $\mathrm{N}$. ulnaris weniger und kleinere, etwa linsengrosse - nur dicht oberhalb der Rinne eine bohnengrosse - Auftreibungen. An der Beugeseite des Vorderarms, deren Form und Farbe durch viel weniger Tumoren verändert ist, als die Streckseite, ist (ausser vereinzelten, zum Theil verödeten Angiomen und 4 Bläschengruppen zwischen oder in unmittelbarer Verbindung mit denselben) nur ein solches farbloses subcutanes Knötchen in der Mittellinie unterhalb der Ellenbeuge und am Ulnaris von der Mitte des Vorderarms abwärts bis $1 \mathrm{~cm}$ oberhalb des Process. styloid. ulnae in grösseren Zwischenräumen 3 solche, auch flache und farblose, aber mehr als erbsengrosse Knoten zu fühlen. In der Vola manus wölben sich an den Aesten des N. median. sowie ulnaris und zwar an dem die Innenseite des Zeige- sowie des Mittelfingers versorgenden Aesten je 2 hinter einander liegende halberbsengross prominirende Knötchen, an dem die Aussenseite des 4. Fingers versorgen- 


\section{7}

den Aste ein kleineres, tiefer liegendes, gleichfalls auf Druck sehr schmerzhaftes Knötchen, nahe der 2. und 4. Interdigitalfalte hingegen je eine weiche, elastische, kugelige, kirchgrosse, auf Druck fast unempfindliche, zuweilen anschwellende und dann sehmerzhafte, venöse Ausbuchtung (Varix); mit der zeitweiligen Intumescenz derselben stellt sich regelmässig Blutaustritt resp. gelbliche Verfärbung an der Rückseite des Basalgelenks des Mittel- resp. kleinen Fingers ein. Auf der Volarseite existiren nur an der 1. und 2. Phalanx des 3. contracturirten und des 4. Fingers, an der 1. sowie an der Aussenseite der 2. Phalanx des kleinen Fingers kleinere, mit Ausnahme der letztgenannten, nicht pigmentirten, hell bräunliche, meistens mit dicker Epidermis bedeckte, nach den Seiten verschiebliche, derbe, auf Druck höchst schmerzhafte Geschwülstchen.

Die Dorsalseite des Vorderarms ist vielfach wellenförmig aufgetrieben durch theils farblose, bis halb-wallnussgrosse, sehr schmerzhafte Tumoren, deren grösste und tiefste, bis an das Periost grenzende vorwiegend an der Radialseite, längs des Radialis oder eines Hautastes desselben, nur kleinere längs der Ulnarseite herabziehen, während auf letzterer mehr die gelblich- oder braunrothen, oberflächlich liegenden schwammigen Angiome überwiegen.

Auf der Mitte des Handrückens erhebt sich, wie oben bemerkt, am stärksten über dem 3. und 4. Metacarpalknochen, nach der inneren Seite allmählich, nach der äusseren etwas jäher abfallend, eine strotzende Anschwellung der oberflächlichen Venen, unter und auch neben welcher sich eine Anzahl kirschkern- bis fast mandelgrosser, harter, höehst schmerzhafter, meistens runder, wie Früchte an $Z$ weigen hängender und genau den Ausstrahlungen der Dorsalnerven aller Finger entsprechender Knoten fühlen lässt. -

An der Dorsalseite der 1. und 2. Phalanx sämmtlicher Finger ausser der 2. des Daumens rundliche resp. längliche, meistens sehr derbe, auf den 1. Phalangen leichter, als auf den 2., wo sie überall grösser sind und hie und $d a$ bis auf's Periost reichen, verschiebliche Tumoren. Diejenigen auf den 1. Phalangen der 2 letzten Finger sind mehr rundliche, flachere, verschiebbare, am Mittelfinger schon tiefer sitzende und verbreiterte, rothe und auf Druck noch empfindliche, noch dünn behaarte Tumoren der Haut und des subcutanen Gewebes, die auf den 2. Phalangen jener und des Zeigefingers sowie auf der 1. des Daumens sitzenden aber länglich, ovoid, oder, wie besonders der höchste auf dem Mittelfinger, der über das 1. Phalangealgelenk weit nach vorn reicht, von höckriger, nach vorn abfallender Form und dunkelbrauner oder Rostfarbe, wie bemerkt, mit dem Periost verwachsen, von gleichmässiger Härte und gegen starken Druck ganz unempfindlich. Doch grenzen an das vordere Ende derjenigen am 3., 4. und an die Aussenseite desjenigen am 5. Finger kleine, linsengrosse, weisse, ebenfalls sehr derbe und indolente Knötehen, deren Gewebe in das der grossen rostfarbigen übergeht, oder vielmehr aus demselben herausgewachsen zu sein scheint. Diese weissen Knötchen will Patient erst 
seit etwa 8 Jahren hinzutreten gesehen haben. Der Tumor am Daumen ist $1,6 \mathrm{~cm}$ lang, $1 \mathrm{~cm}$ hoch, der am Zeigefinger ebenso lang und $\frac{1}{2} \mathrm{~cm}$ hoch, am Mittelfinger $2 \mathrm{~cm} 9 \mathrm{~mm}$ lang und $1,2 \mathrm{~cm}$ hoch, am 4. Finger $2 \mathrm{~cm}$ lang und $9 \mathrm{~mm}$ hoch, am kleinen $1,8 \mathrm{~cm}$ lang und $2 \mathrm{~mm}$ hoch. Endlich liegt je ein ganz isolirtes farbloses, auf Druck schmerzhaftes Knötchen auf der 1. Phalanx des Zeigefingers an der Radialseite, einwärts, aber getrennt von einem länglichen braunen Fibrom, sowie an der Basis der Aussenseite des kleinen Fingers.

Obwohl uns keinerlei Excision jener Fingertumoren behufs anatomischer Untersuchung gestattet ist, weil vor $10 \mathrm{Jahren}$ nach jeder Electropunctur der Angiome am Vorderarm äusserst heftige Reaction, Anschwellung des Armes und starkes Fieber bis zu 14 Tagen eintrat, dürfen wir dieselben doch nach dem im Vorstehenden nachgewiesenen Befunde so zahlreicher Neurome im ganzen Ausbreitungsbezirk des Plexus brachialis sinister, von den Stämmen und Zweigen, sogar den thoracischen Aesten, bis in die Hautäste der Hand hinab und im Anschluss an den anatomischen Nachweis des genetischen Zusammenhanges zwischen multiplen Neuromen und multiplen Fibromen der Haut, welchen v. Recklinghausen ${ }^{1}$ ) in seiner vortrefflichen Monographie unlängst an 2 Fällen von universellem congenitalen Fibroma molluscum geführt hat, für neuromatöse Fibrome oder Neurofibrome erklären, d. h. für „Fibrome, welche in kleinen Cutisnerven nach dem Typus der Fibrome in den Nervenstämmen mit Verlagerung, aber anfänglicher Erhaltung der Primitivnervenfasern gebildet sind", in deren peripherischsten aber die Nervenfasern durch die massenhafte und gleichmässige fibrilläre resp. Fibromwucherung wahrscheinlich erdrückt worden sind ${ }^{2}$ ). -

Um den Befund an den Gefässen dieser Extremität zu vervollständigen, ist noch zu bemerken, dass die rechte Arter. axillar., brachialis, sowie radialis von"grösserem Umfange und stärkerer Welle sind, als die der linken Seite. Am Herzen ist nichts Abnormes nachzuweisen.

1) Ueber die multiplen Fibrome der Haut und ihre Beziehungen zu den multiplen Neuromen. Berlin 1882.

${ }^{2}$ ) Uebrigens bildet v. Recklinghausen auf Taf. V, Fig. 9 Fibromgewebe aus einem viel kleineren, von Blutgefässen durchzogenen Fibrom des Periostes der Tibia ab (Fall I, S. 6), in dem noch ein axiales Nervenfaserbündel erhalten war. 
Alle Bewegungen des Armes sind möglich, ausser Supination des Vorderarms, die dem Patienten nur theilweise gelingt. Die Fingerbeugung kann nicht ganz bis zur Schliessung einer Faust, die Beugung im Daumenwurzelgelenk gar nicht - überall wegen Druckes kleiner Neuromknötchen bei den intendirten Bewegungen - vollbracht werden. Passive Bewegungsversuche in jenem Gelenk sowie behufs vollständiger Supination des Vorderarms schmerzen. Die galvanische und faradische Reaction aller Muskeln dieser Extremität von denjenigen des Schulterblatts und dem besonders stark abgemagerten Deltoideus an bis zu den Mm. lumbricales der Finger ist sowohl bei directer als bei Nervenreizung normal, desgleichen die Sensibilität. Spontane Schmerzen existiren nicht, ausserordentlich starke aber, wie schon öfter bemerkt, auf Druck aller Neuromknoten. Hauptsächlich zur Vermeidung desselben hat Pat. von der ersten Kindheit an diese Extremität möglichst wenig in Action versetzt, wie er sie auch jetzt lieblingsweise schlaff herabhängen lässt, und dieser Inactivität ist ein hervorragender Antheil an dem Schwund der Muskeln und zum Theil und indirect an dem geringeren, wenigstens Dickenwachsthum auch einiger Knochen dieser Seite, sowie an der secundären Scoliose und der compensirenden Lordose durch überwiegenden Muskelzug nach rechts zuzuschreiben. Für das Zurückbleiben der Knochen im Längenwachsthum jedoch, wie es die nachfolgenden Ziffern veranschaulichen, sind wohl zuoberst die zahlreichen Bildungsanomalien an den Nerven und die schwächere Entwickelung der Arterie dieser Seite in's Auge zu fassen. - Von sogenannten trophischen Störungen sehen wir noch, dass die Haut der Streckseite des linken Vorderarms und des äusseren Drittels der linken Hand stärker behaart ist, als rechts (bei gleichem Haarwuchs in beiden Achseln). Die $\mathrm{Nägel}$ wachsen normal, nur die Nagelbetten des linken Daumens und Zeigefingers sind stärker gewölbt und ein wenig cyanotisch ${ }^{1}$.

Die linke Hand sowie Achsel schwitzt viel leichter und reichlicher, als die rechte. Auch ist sie um ungefähr $1^{\circ} \mathrm{C}$. wärmer. -

Zum Schluss endlich mögen einige Messungsresultate ziffermässig das Zurückbleiben der einzelnen Theile dieser Extremität in der Entwickelung veranschaulichen.

Der linke Oberarmumfang beträgt in der Mitte 17,5, der rech te $22 \frac{1}{2} \mathrm{~cm}$. Unterarmumang - $\quad-\quad$ - $17,5, \quad-\quad-20$ -

1) Es ist mir wahrscheinlich, dass dieses Verhalten einfach aus dem, durch einige der tieferen, bis auf's Periost reichenden Tumoren an der Radialseite des Vorderarms noch erschwerteren venösen Rückfluss aus diesen 2 Fingern und nicht aus Nerveneinfluss resultirt, weil ich bei einem 9jährigen Knaben, an welchem Prof. Heinrich Jacobson eine Stenose der Art. pulmonal. diagnosticirt hatte, an sämmtlichen Nagelbetten der Finger und der Zehen dieselbe Wölbung nebst stärkerer Cyanose constatirt habe. 
Die Knochendicken, $15 \mathrm{~cm}$ über dem Olecranon mit dem fest an den Knochen gedrückten Tasterzirkel gemessen, betragen für:

$$
\text { den linken Humerus } \mathbf{2 , 5} \text {, den rechten } \mathbf{3 , 9} \mathrm{cm} \text {, }
$$

während diejenigen der Vorderarmknochen, $5 \frac{1}{2} \mathrm{~cm}$ über den Process. styloid. an geschwulstfreien Stellen gemessen, gleich sind:

$$
\begin{aligned}
& \text { der linke Radius } 2,0 \text {, der rechte } 2,0 \mathrm{~cm} \text {, } \\
& \text { die linke Ulna, 2,2, die rechte } 2,2 \text { - }
\end{aligned}
$$

Längenmaass $\theta$ :

Distanz vom Acromion bis Olecranon links 33,5 , rechts $36 \mathrm{~cm}$,

Länge der Ulna (Olecranon bis Process. styloid.) 23, rechts 24 Handlängenmaasse (mittelst Zirkels festgestellt):

Vom Process. styloid. Ulnae bis zur Spitze

des kleinen Fingers: . . . . . links $10 \frac{1}{2}$, rechts $13 \mathrm{~cm}$.

Von der Mitte des Handgeleriks bis zur

Mittelfingerspitze . . . . . . . - 15 - 18 -

Vom Proc. styloid. Radii bis zur Spitze des

Daumens . . . . . . . . . - 11 - 12 -

Länge des kleinen Fingers . . . . - $6 \frac{1}{2}-7$ -

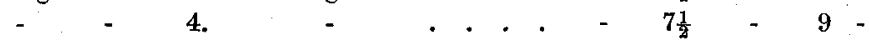

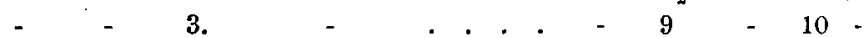

- - 2. $\quad$ - $\quad$. . . . $-8 \frac{1}{2}-9$ -

- - Daumens . . . . . . - 5 $5 \frac{3}{4}-6 \frac{3}{4}-$

- desselben bis zur Basis Metacarpi 1 - 9 - $10 \frac{1}{2}$ -

Die Breite der Handwurzel zwischen

Process. styloid. Ulnae et Radii . . . - $\quad 5 \quad-\quad 5 \frac{3}{4}-$

Breite der Hand zwischen Basalgelenk des

Daumens und kleinen Fingers . . - $8 \frac{1}{2}-10 \frac{3}{4}-$ 